\title{
Is it important to differentiate between peri-procedural myocardial injury and persistent myocardial scar?
}

\author{
Margaret McEntegart $^{1,2}$, David Corcoran ${ }^{1,2}$, Colin Berry $^{1,2}$ \\ ${ }^{1}$ West of Scotland Heart and Lung Centre, Golden Jubilee National Hospital, Clydebank, UK; ${ }^{2}$ British Heart Foundation Glasgow Cardiovascular \\ Research Centre, Institute of Cardiovascular and Medical Sciences, University of Glasgow, Glasgow, UK \\ Correspondence to: Margaret McEntegart, PhD. Golden Jubilee National Hospital \& University of Glasgow, Agamemnon St, Glasgow G81 4DY, UK. \\ Email: margaret.mcentegart@nhs.net. \\ Provenance: This is an invited article commissioned by the Section Editor Hui-Ping Zhang (Department of Cardiology, Beijing Hospital, the Fifth \\ Affiliated Hospital of Peking University, Beijing, China). \\ Response to: Januszek R, Bartuś S. Does the use of rotational atherectomy procedure during percutaneous coronary interventions influence the \\ frequency of procedure-related myocardial injury assessed by cardiac magnetic resonance? J Thorac Dis 2018;10:S3050-2. \\ Jinnouchi H, Sakakura K, Fujita H. Periprocedural myocardial infarction is all the same? J Thorac Dis 2018;10:S3176-81.
}

Submitted Oct 30, 2018. Accepted for publication Nov 16, 2018.

doi: $10.21037 /$ jtd.2018.11.84

View this article at: http://dx.doi.org/10.21037/jtd.2018.11.84

With the growth of complex high-risk percutaneous coronary intervention (PCI) it is of increasing importance to be able to quantify procedural risk and describe the consequence of peri-procedural complications. With the increasing prevalence of calcific coronary artery disease (CAD) there is a growing need for adjunctive calcium modifying techniques, with rotational atherectomy (RA) the most commonly used method. In this context, we conducted a cohort study of patients having PCI with RA to determine the incidence of peri-procedural myocardial infarction (PMI), perceived to be the most frequently associated complication. We applied the two current definitions of PMI, the $3^{\text {rd }}$ universal definition $\left(3^{\text {rd }}\right.$ UD) and Society for Cardiovascular and Interventions (SCAI) definition. Cardiac magnetic resonance (CMR) imaging is the reference standard method for myocardial tissue characterization and myocardial infarct assessment. We therefore used CMR imaging to assess the accuracy of each definition of PMI, and to determine the mid-term persistence of any detected myocardial injury (1). The multiparametric CMR protocol included cine imaging to detect new regional wall motion abnormalities (WMA), parametric T2 mapping for acute myocardial oedema, and late gadolinium enhancement (LGE) imaging to detect myocardial scar.
We would like to thank Januszek et al. and Jinnouchi et al. for their review of the wider literature and interesting commentary on the study. As stated by Januszek et al. (2), Lim et al. previously reported that CK-MB defined type 4a MI correlated better with CMR detected infarct, while cardiac troponin was over-sensitive (3). Despite this, with the wide adoption of high-sensitivity troponin in the diagnosis of MI, and with CK-MB no longer routinely available, we elected to use troponin to facilitate translation of the data directly into clinical practice.

A troponin $>5 \times$ upper reference limit (URL) was detected in $68 \%$ of our cohort. On applying the $3^{\text {rd }}$ UD (also requiring $>20$ mins of chest pain, ECG ST-segment changes or new $\mathrm{Q}$ waves, angiographic deterioration in flow, or imaging evidence of loss of viable myocardium or new wall motion abnormality), the incidence of type 4a MI was $10 \%$. With the addition of CMR imaging the incidence of PMI was $24 \%$, i.e., CMR was able to detect acute myocardial injury in an additional $14 \%$ of patients which was not apparent on the post-procedural ECG or echocardiogram. The prevalence of PMI decreased to $14 \%$ on the 6-month follow-up CMR. Contrary to the comment by Januszek et al., and as acknowledged by Jinnouchi et al., we had anticipated the incidence of PMI would increase 
with the addition of the CMR data to the $3^{\text {rd }} \mathrm{UD}$, as ECG and echocardiography are known to have limited sensitivity particularly for small subendocardial infarcts $(2,4)$.

As commented by Jinnouchi et al., the comparison of the day 7 and 6-month CMR data, showing complete resolution of myocardial oedema and new WMAs but persistence of LGE, highlights the difference between transient myocardial injury (i.e., myocardial stunning) and persistent myocardial scar. This draws attention to the potential issue in analyses by other groups, which by including all $3^{\text {rd }}$ UD type 4a MI, combined patients with better and poorer prognoses into one cohort.

We agree with Jinnouchi et al. that our data does not support routine usage of CMR imaging for the diagnosis of PMI, while its prediction of clinical outcomes remain unclear. In particular, the prognostic relevance of CMR detected LGE and its use to stratify patients with $3^{\text {rd }}$ UD PMI requires further investigation.

\section{Acknowledgements}

Funding: The British Heart Foundation has supported DC (FS/14/15/30661) and CB (RE/13/5/30177; FS/14/15/30661; FS172632744; PG-17- 25-32884).

Cite this article as: McEntegart $M$, Corcoran D, Berry C. Is it important to differentiate between peri-procedural myocardial injury and persistent myocardial scar? J Thorac Dis 2018;10(12):E830-E831. doi: 10.21037/jtd.2018.11.84

\section{Footnote}

Conflicts of Interest: The authors have no conflicts of interest to declare.

\section{References}

1. McEntegart M, Corcoran D, Carrick D, et al. Incidence of procedural myocardial infarction and cardiac magnetic resonance imaging-detected myocardial injury following percutaneous coronary intervention with rotational atherectomy. EuroIntervention 2018;14:819-23.

2. Januszek R, Bartuś S. Does the use of rotational atherectomy procedure during percutaneous coronary interventions influence the frequency of procedure-related myocardial injury assessed by cardiac magnetic resonance? J Thorac Dis 2018;10:S3050-2.

3. Lim CC, van Gaal WJ, Testa L, et al. With the "universal definition," measurement of creatine kinase-myocardial band rather than troponin allows more accurate diagnosis of periprocedural necrosis and infarction after coronary intervention. J Am Coll Cardiol 2011;57:653-61.

4. Jinnouchi H, Sakakura K, Fujita H. Peri-procedural myocardial infarction is all the same? J Thorac Dis 2018;10:S3176-81. 